\title{
Influence of Multi Stenosis on Hemodynamic Parameters in an Idealized Coronary Artery Model
}

\author{
S. Kamangar $^{\dagger}$ \\ Mechanical Engineering Department, College of Engineering, King Khalid University, Abha, 61421, \\ Kingdom Saudi Arabia \\ †Email: sarfaraz.kamangar@gmail.com
}

(Received January 18, 2021; accepted July 16, 2021)

\begin{abstract}
This study explores the influence of tandem stenosis on hemodynamic constraints in idealized coronary artery models with different interspace distance between the stenosis and its severity. The blood was assumed as nonNewtonian, incompressible, and pulsatile fluid. The hemodynamic parameters of blood, such as the oscillatory shear index (OSI), wall shear stress (WSS) and time average wall shear stress (TAWSS) are obtained and compared for various degrees of stenosis and interspacing distance in various blood models. The computed results showed that as the interspacing distance between the stenosis decreases, the low wall shear stress area increases for the model P70_D70, leading to further progression of stenosis in the distal region. No significant variation was observed for the model P70_D90, whereas the low WSS region increases as the interspace distance of proximal and distal stenosis increases for the model P90_D70. The highest TAWSS sites were created across the $90 \%$ AS (area stenosis) for all the cases studied. As the higher value of TAWSS is clinically significant since it could damage the endothelial layer. It is well known that the maximum value of OSI is strongly associated with the critical areas of stenosis rupture. The maximum value of OSI was found at the proximal and distal stenosis for all the models simulated.
\end{abstract}

Keywords: Wall shear stress; Computational fluid dynamics; Non-Newtonian; Endothelial layer.

\section{NOMENCLATURE}

\begin{tabular}{|c|c|c|c|}
\hline \multicolumn{2}{|c|}{ Symbols } & \multirow{2}{*}{$\begin{array}{l}\mu \\
\tau\end{array}$} & \multirow{2}{*}{$\begin{array}{l}\text { viscosity } \\
\text { viscous stress tensor }\end{array}$} \\
\hline$a$ & radius of an artery & & \\
\hline$d$ & diameter & $\lambda$ & relaxation time \\
\hline$h$ & $\begin{array}{l}\text { the maximum projection of the stenosis into } \\
\text { the lumen }\end{array}$ & $\mu_{0}$ & viscosity at zero shear rate \\
\hline$n$ & power index & $\mu_{\infty}$ & viscosity at infinite shear rate \\
\hline$\tilde{\eta}(\tilde{z})$ & radius of stenosis & $\gamma$ & shear rate \\
\hline$P$ & pressure & & \\
\hline$p(t)$ & transient Pulsatile pressure & \multicolumn{2}{|c|}{ Abbreviations } \\
\hline$t$ & time & WSS & Wall Shear Stress \\
\hline $\bar{u}$ & time dependent parabolic velocity & TAWSS & Time Average Wall Shear Stress \\
\hline $\bar{u}_{p-t}$ & $\begin{array}{l}\text { normalized velocity with respect to the peak } \\
\text { temporal velocity }\end{array}$ & OSI & Oscillatory Shear Index \\
\hline$v$ & $3 \mathrm{D}$ velocity vector & $A S$ & Area Stenosis \\
\hline \multirow[t]{2}{*}{$\tilde{z}$} & along the axis of the artery & $L D L$ & Low-Density Lipoprotein \\
\hline & & CCTA & $\begin{array}{l}\text { Coronary Computed Tomography } \\
\text { Angiography }\end{array}$ \\
\hline \multicolumn{2}{|c|}{ Greek Symbols } & $M R I$ & Magnetic Resonance Imaging \\
\hline$\rho$ & density & $C F D$ & Computational Fluid Dynamic \\
\hline
\end{tabular}




\section{INTRODUCTION}

The most common form of cardiovascular disease is Atherosclerosis, in which the lumen diameter of a coronary artery reduces due to the deposition of lowdensity lipoprotein (LDL). This results an insufficient oxygenated blood flow to the heart muscles resulting in infarction or myocardial ischemia or necrosis or more severely. The progression and development of stenosis originate across the bifurcation, branches, and curvatures of the coronary artery (Berger and Jou 2000); Caro 1969). Many studies reported a strong association between local hemodynamic factors such as wall shear stress, flow pattern, and endothelial cell functioning (Malek et al. 1999; Stone et al. 2003). Despite the huge advancements in the current visualization medical imaging technique, such as coronary computed tomography angiography (CCTA) and magnetic resonance imaging (MRI), it remains challenging to predict the local disturbed flow pattern noninvasively. These imaging techniques are limited to determine the alteration in morphology of arteries and can detect the formation of the plaques with high accuracy. The low wall shear stress plays a key role in the formation of atherosclerosis reported by numerous studies (Chatzizisis et al. 2007). In the blood vessels, stenosis geometry may play an important role in the formation of recirculation or flow disturbances at the post stenosis. Several studies demonstrated that the arteries with mild stenosis could produce the transitional flow to turbulence. CFD studies have been used to predict the atherosclerotic plaque progression and disturbed flow behavior in the last few years by many researchers (Badruddin et al. 2019; Kamangar et al. 2017b; Karimi et al. 2014). Numerous studies conducted in which the blood flow behavior was assumed as laminar (Frauenfelder et al. 2007; Wu et al. 2015), and some studied non-Newtonian effects of blood and turbulence transition in idealized stenosed artery with single stenosis (Li et al. 2007; Moreno and Bhaganagar 2013; Nosovitsky et al. 1997). Many studies reported that, the pulsatile flow through a series of stenosed vessel. It was reported that the pressure drop across multiple stenoses is sum of the individual stenoses pressure drops. They also observed that interspacing distance between stenosis leads to minor pressure drop (Karayannacos et al. 1977; Rathish Kumar et al. 2002). In the current study, the hemodynamic descriptors around the proximal and distal stenoses in an idealized coronary artery model with various stenosis severity and interspacing distance are investigated using computational fluid dynamics. The oscillatory shear index wall shear stress, time average wall shear stress, were compared to recognize the regions of progression and development of stenosis and rupture of arterial behind the proximal stenosis and around the distal stenosis and vice versa.

\section{METHODOLOGY}

\subsection{Idealized coronary artery model}

Figure 1(b) shows the multi stenosed computational blood domains of an idealized coronary artery model of P70_D70, constructed with varying stenosis severity having different interspacing distance by using Ansys CFX (ANSYS, Canonsburg, PA, USA). It consists of an axisymmetric straight cylindrical tube. The percentage area of stenosis (AS) was calculated by using Eqs. 1. The axisymmetric stenosed models in series was constructed by using by the following Eqs. 2 (Dash et al. 1999; Kamangar et al. 2017a; 2014).

$$
\begin{gathered}
\text { Area stenosis (AS)\% }=\frac{\left(\pi \times a^{2}\right)-\left[\pi \times(a-h)^{2}\right]}{\pi \times a^{2}} \\
\frac{\tilde{\eta}(\tilde{z})}{a}=1-\frac{h}{a} \sin \pi\left(\frac{\tilde{z}-d}{L}\right), \\
d \leq \tilde{z} \leq d+L,
\end{gathered}
$$

The lumen diameter of an idealized coronary artery model is 3mm and the length of stenosis has been considered as $10 \mathrm{~mm}$ in all models. The interspacing distances between the proximal (P) and distal(D) stenosis are $3 \mathrm{~mm}, 6 \mathrm{~mm}$, and $10 \mathrm{~mm}$ considered. The varying stenosis severity [proximal and distal area stenoses with 70\% AS (P70_D70), proximal 70\% AS, distal 90\%AS (P70_D90) and proximal 90\% AS, distal 70\% AS (P90_D70)] and interspacing distance (3mm, 6mm, and $10 \mathrm{~mm}$ ) are simulated. The ANSYS ICEM CFD was used to mesh the computational models with hexahedral elements. The unstructured hexahedral elements across the stenosis and inlet surface are generated as shown in Fig. 1 (a) and (c), respectively. The mesh independent study was performed to determine the best results and to minimize the cost and time of computer simulation. Coarse, medium, and fine mesh with 528865, 748804 , and 989874 elements, respectively were created. Figure 2a shows the various numbers of meshes were plotted for the pressure drop along the length of the artery. As observed from the Fig. 2a, less the $0.5 \%$ errors of the solutions between different meshes was found so the mesh element with 748804 was used to reduce computational time and cost.

\subsection{Model Verification}

To verify the solution of the current study, the results were compared with the previously published results (Jahangiri et al. 2015) as shown in Fig. 2b. The results of pressure drop along the length of the artery demonstrate a better match with the results of previously published articles (Jahangiri et al. 2015). Whereas there is a slight difference in results between the current study and the simulation results of previously published articles, this is mainly due to the degree of stenosis (P70_D70) was considered in this study, whereas the degree of stenosis P80_D80 was used in the published articles.

\subsection{Blood flow modeling}

The blood is assumed to follow Bird-Carreau shear thinning non-Newtonian, incompressible flow model as below (Kamangar et al. 2017a).

$\rho\left(\frac{\partial v}{\partial t}+v \cdot \nabla v\right)=\nabla . \tau-\nabla P$

$\nabla \cdot v=0$ 


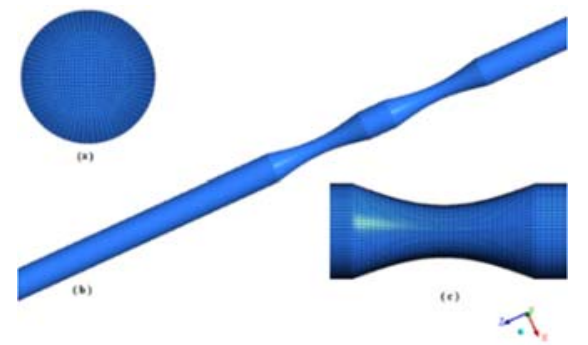

Fig. 1: Computational meshed model.

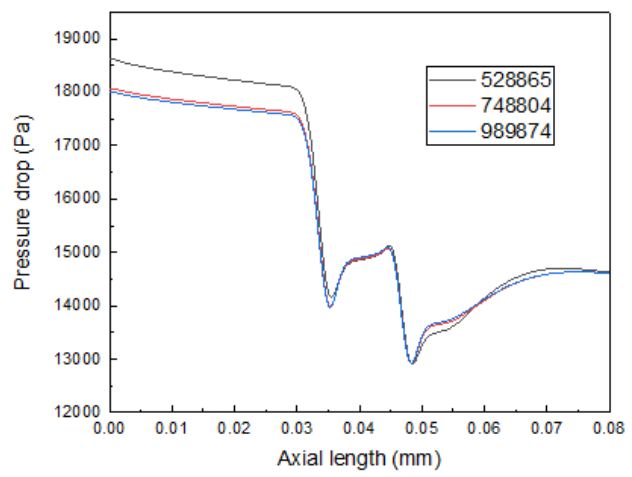

Fig. 2a Mesh independent study.

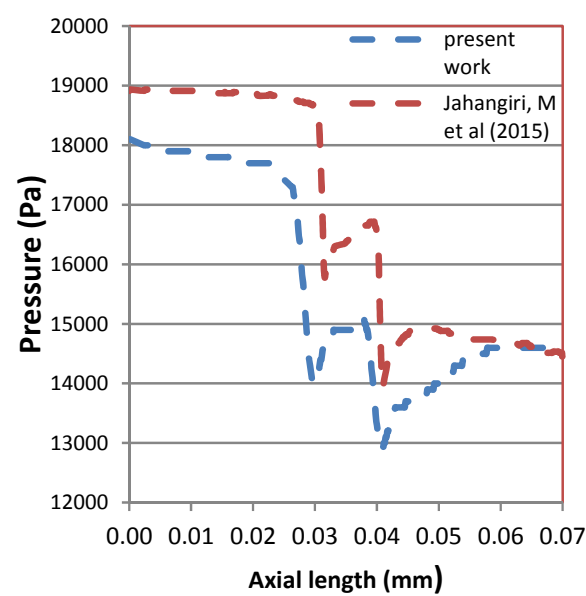

Fig. 2b: Comparison of present study with previously published results.

$\mu=\mu_{\infty}+\left(\mu_{0}-\mu_{\infty}\right)\left[1+(\lambda \gamma)^{2}\right]^{(n-1) / 2}$

The density is assumed as $1050 \mathrm{~kg} / \mathrm{m}^{3}$ (Kamangar et al. 2019). The simulations study was performed by using ASYS CFX 2020R1 (ANSYS Inc). The boundary conditions at inlet and outlet applied as shown in Fig. 3a and 3b respectively with no-slip wall boundary condition.

\subsection{Wall Shear Stress (WSS)}

The WSS plays a vital role in the formation of atherosclerotic plaque on the artery walls. The WSS is a tangential force that acts on the wall of the artery contains the endothelial layers can be calculated as follows

$\tau=\mu\left(\frac{\partial \mathrm{u}}{\partial \mathrm{y}}\right)_{\mathrm{W}}$

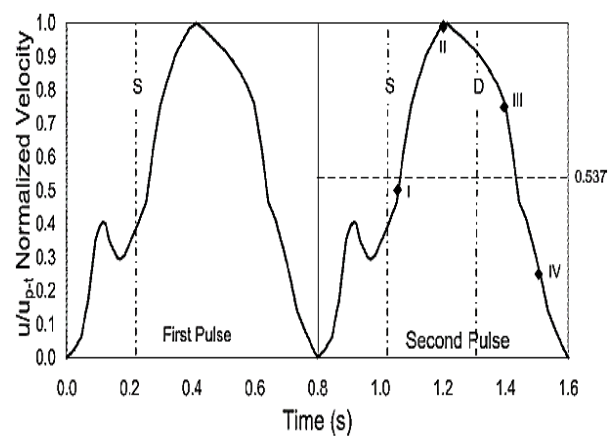

(a)

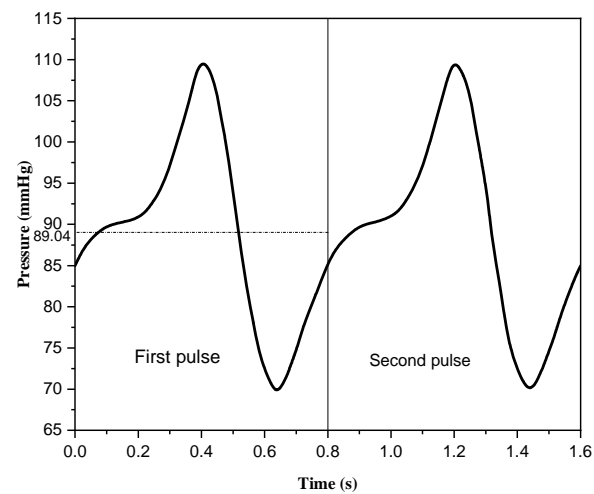

(b)

Fig. 3: (a) Physiological coronary flow wave form applied at inlet. (b) Imposed outlet pressure (Banerjee et al. 2003; Konala et al. 2011; Govindaraju et al. 2016).

\subsection{Time-Averaged Wall Shear Stress (TAWSS)}

The TAWSS over one cardiac cycle is known as time average wall shear stress. The TAWSS can be calculated as

TAWSS $=\frac{1}{\mathrm{~T}} \int_{0}^{\mathrm{T}}|\mathrm{WSS}| \mathrm{dt}$

\subsection{Oscillatory Shear Index (OSI)}

It is a hemodynamic variable used to calculate the deviations in the direction of the WSS vector throughout the cardiac cycle It can be calculated as

$o S I=0.5 \times\left(1-\frac{\left|\int_{0}^{\tau} \mathrm{WSSdt}\right|}{\int_{0}^{\tau}|\mathrm{WSS}| \mathrm{dt}}\right)$ 


\section{RESULTS}

\subsection{Influence of interspacing distance and stenosis severity on WSS}

The effect of interspacing distance and degree of stenosis severity present in series on wall shear stress was represents in Figs. 4, 5, and 6 for systolic phase of cardiac cycle. Several studies reported that the risk of progression and development of plaque is higher, whenever the inner surface of an artery is subjected to a low wall shear stress. In order to determine the locations prone to the development of clinical atherosclerotic diseases, wall shear stress contours are plotted for a range of 0-5 Pa for the systolic phase. It is clearly seen from Fig. 4, that a larger area of low wall shear stress was observed for the model with interspacing distance is $3 \mathrm{~mm}$. The minimum wall shear stress in the range $0-0.56 \mathrm{~Pa}$ was found distal to the $70 \%$ AS stenosis. A low wall shear stress region after the distal stenosis decreases as the interspacing distance between the stenosis is increased from $6 \mathrm{~mm}$ to $10 \mathrm{~mm}$. The low wall shear stress regions are observed in the distal section which can be attributed to low velocity. No significant differences were observed for the model P70-D90,

irrespective of the interdistance among proximal and distal stenosis. For the model P90-D70, a low wall shear stress was visible immediately after the stenosis, and its area of low wall shear stress increases with the increase in the distance between the stenosis.

\subsection{Effect of interspacing distance and stenosis severity on TAWASS}

The average wall shear stress over one cardiac cycle is known as time average wall shear stress. Figures 7 , 8, and 9 show the effect of interspacing distance and stenosis severity on TAWSS. The highest TAWSS sites were found at the stenosis regions. These higher areas of TAWSS clinically significant as the high values of TAWSS can lead to harm the endothelial layer present on the inner surface of the lumen (Suess et al. 2016). The low TAWSS regions in the range 0 to 0.02 were found between the proximal and distal stenosis and immediately after the stenosis. The low TAWSS area after the stenosis decreases with the increase in the two stenosis distance for the model P70-D70 and P70-D90. The low TAWSS area after the stenosis increases due to decreased stenosis distance for the model P90-D70.

\subsection{Impact of interspacing distance and stenosis severity on OSI}

Past few decades observed the studies on blood flow in arteries revealed that the high magnitude of OSI and low WSS are strongly associated with the critical areas of stenosis rupture and stenosis formation, respectively (He and Ku 1996). Figure 10, 11 , and 12 describes the distribution of OSI contours

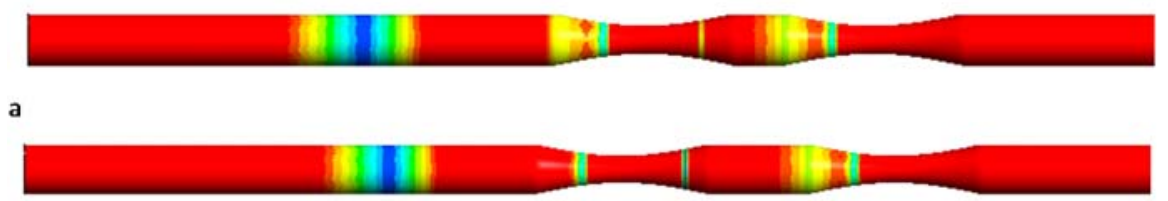

b

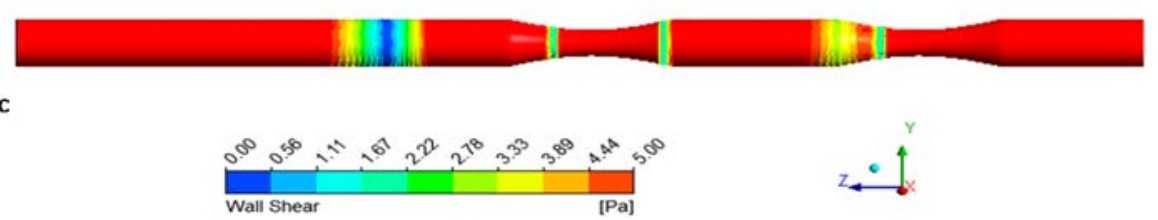

Fig. 4: WSS P70_D70 with interspacing distance for systole cardiac cycle a) $3 \mathrm{~mm}$ b) $6 \mathrm{~mm}$ c) $10 \mathrm{~mm}$.

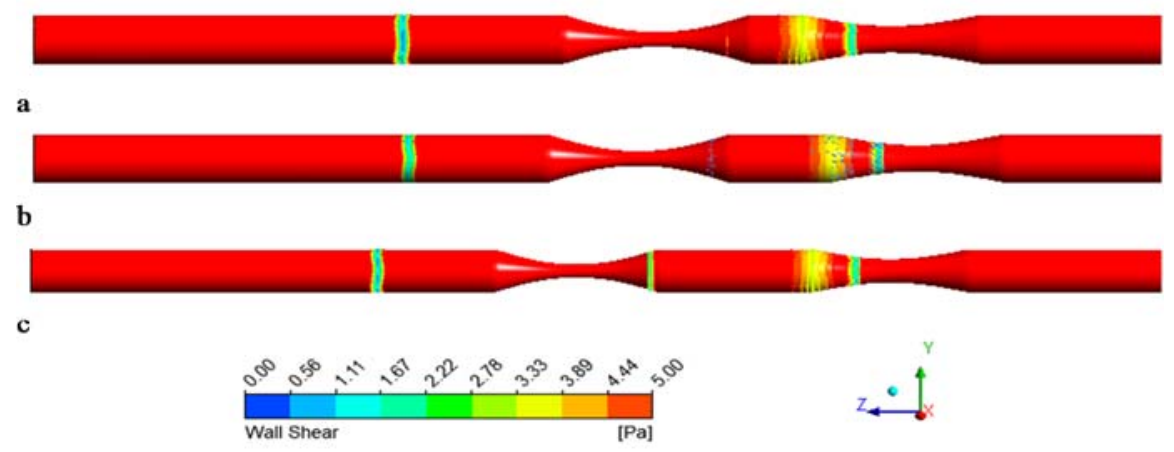

Fig. 5: WSS for P70_D90 with interspacing distance for systole cardiac cycle a)3mm b) $6 \mathrm{~mm}$ c) $10 \mathrm{~mm}$. 


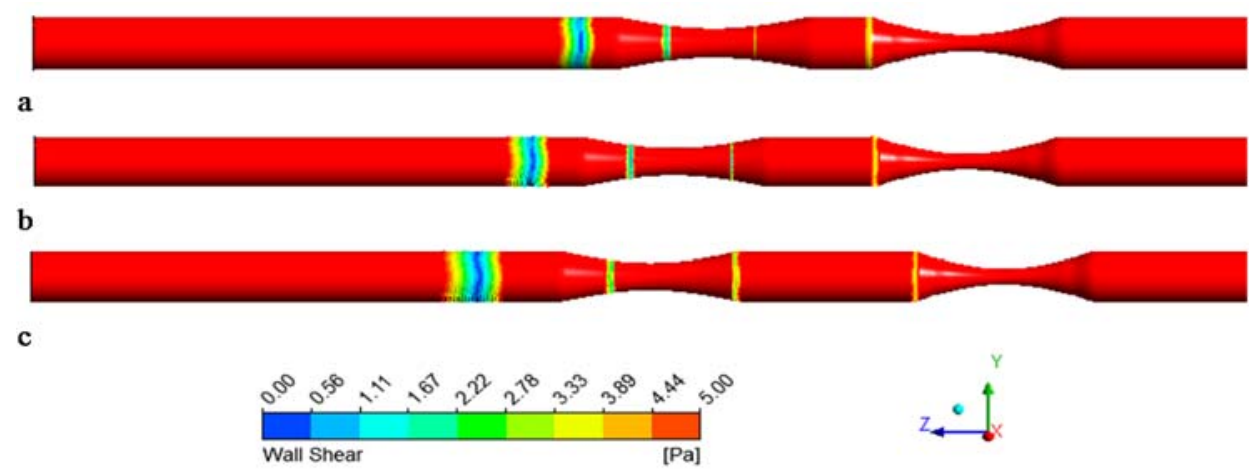

Fig. 6: WSS for P90_D70 with interspacing distance for systole cardiac cycle a) $3 \mathrm{~mm} \mathrm{b)} 6 \mathrm{~mm} \mathrm{c)} 10 \mathrm{~mm}$.

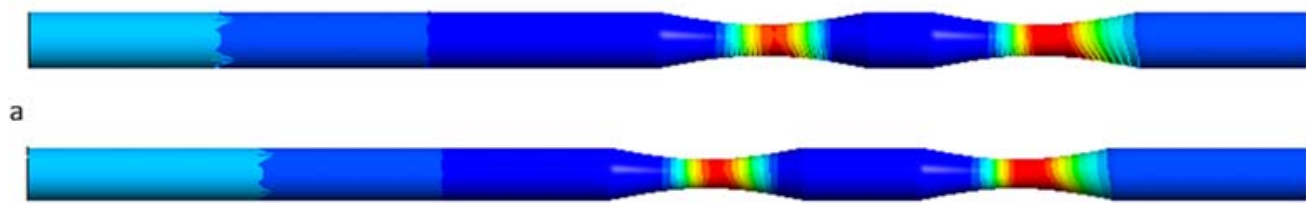

b
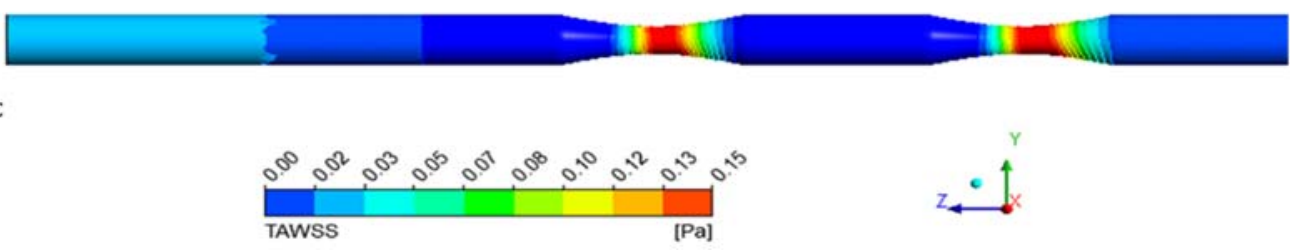

Fig. 7: TAWSS for P70_D70 with interspacing distance for systole cardiac cycle a) $3 \mathrm{~mm}$ b) $6 \mathrm{~mm}$ c) $10 \mathrm{~mm}$.

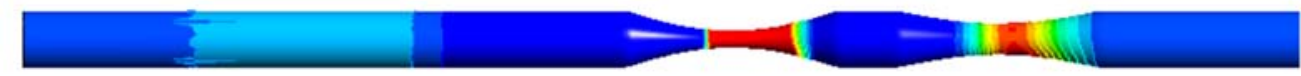

a

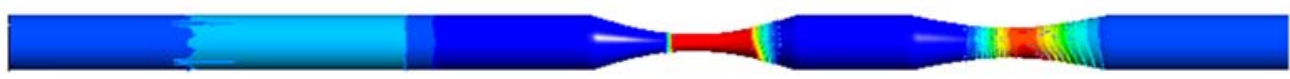

b

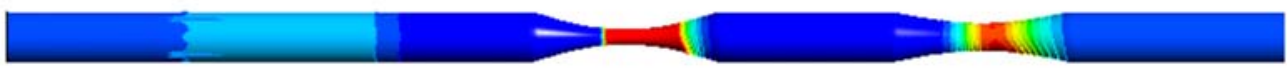

c
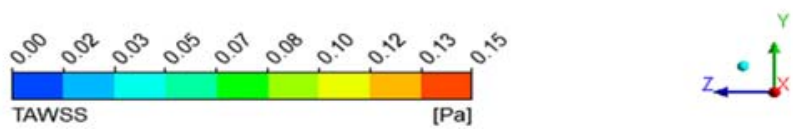

Fig. 8: TAWSS for P70_D90 with interspacing distance for systole cardiac cycle a) 3mm b) 6mm c) $10 \mathrm{~mm}$.

for various stenosis severity and interspacing distance on the artery wall during a single pulsatile period. It is well-known fact that the risk of rupture of stenosis is higher and causes sufficient endothelial layer damage when the inner surface is exposed to higher OSI than the physiological levels of OSI. The higher OSI area was observed at the stenosis and distal to the stenosis. A slightly higher area of OSI was noted across the $90 \%$ AS as compared to $70 \%$ AS. A slight increase in the OSI area distal to the stenosis was reported as the distance between the stenosis increases for the model P90_D70. 


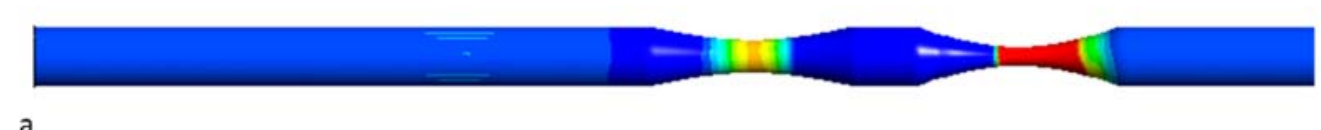

a

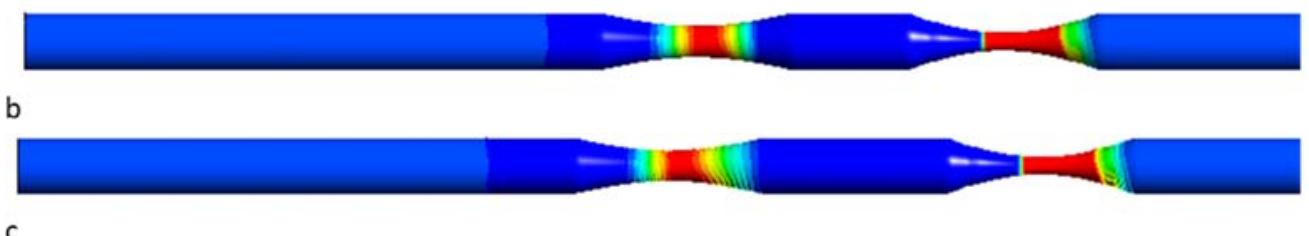

C

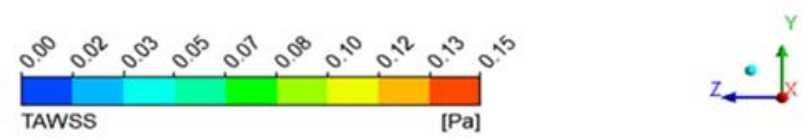

Fig. 9: TAWSS for P90_D70 with interspacing distance for systole cardiac cycle a) $3 \mathrm{~mm} \mathrm{b)} 6 \mathrm{~mm}$ c) $10 \mathrm{~mm}$.

a

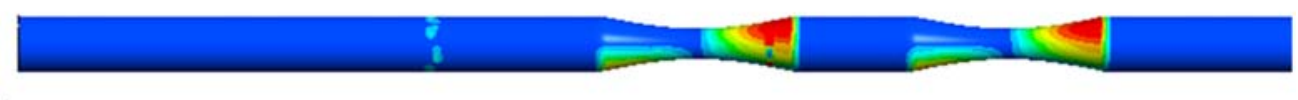

b

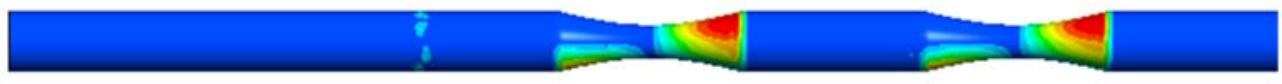

C
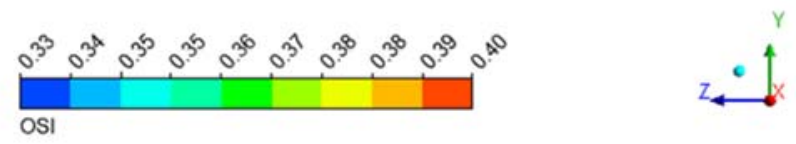

Fig. 10: OSI for P70_D70 with interspacing distance for systole cardiac cycle a) $3 \mathrm{~mm}$ b) $6 \mathrm{~mm}$ c) $10 \mathrm{~mm}$.

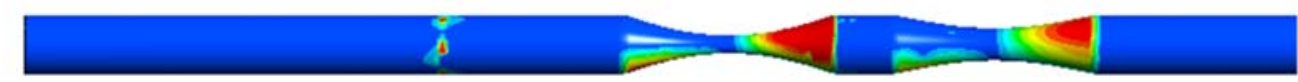

a

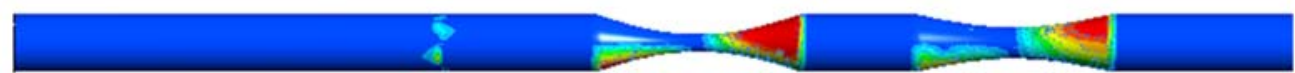

b

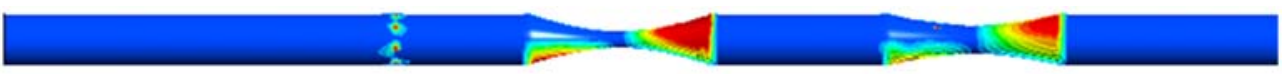

C

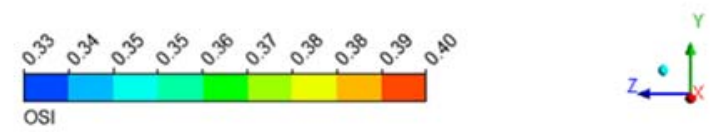

Fig. 11: OSI for P70_D90 with interspacing distance for systole cardiac cycle a) $3 \mathrm{~mm}$ b) $6 \mathrm{~mm}$ c) $10 \mathrm{~mm}$. 

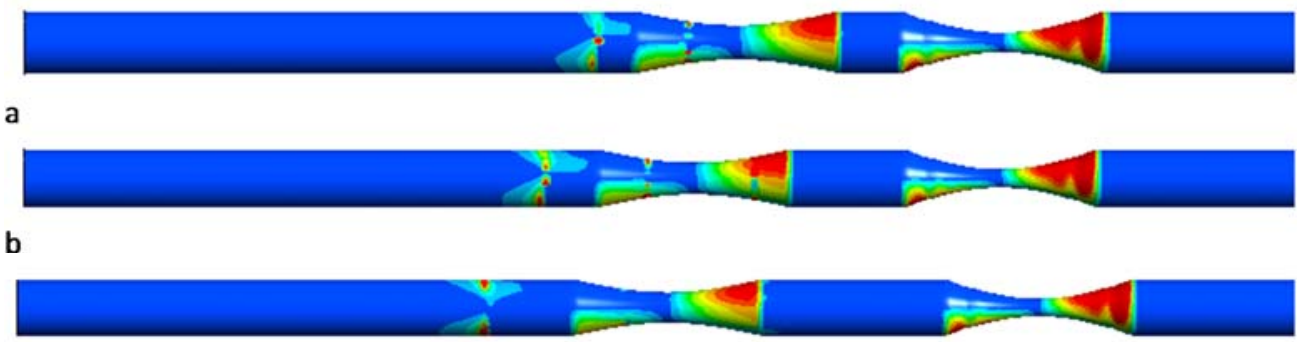

C
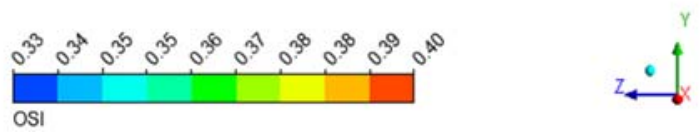

Fig. 12: OSI distributions in idealized coronary artery model for P90_D70 with interspacing distance for systole cardiac cycle a) $3 \mathrm{~mm}$ b) $6 \mathrm{~mm}$ c) $10 \mathrm{~mm}$.

\section{Discussion}

The current study investigates the influence of stenosis severity and interspacing distance between the stenosis in coronary arteries on hemodynamic variables, Time average wall shear stress, and Oscillatory shear index. A significant effect of stenosis severity and interspacing distance was observed on these hemodynamic parameters. The variations in the wall shear stress-based hemodynamic parameters were computed for multi stenosis models with different interspacing distances. Figures 4, 5, and 6 show the wall shear stress area having less than $5 \mathrm{~Pa}$ increases with the increases in distance between the stenosis for the models P70_D70 and P90_D70, and it reduces considerably for the model P70_D90. This may be because the flow of blood for the model P70_D90 after the $90 \%$ AS behaves like a strong jet with higher velocity after the severe stenosis and high wall shear stress observed. According to the previous studies (Malek et al. 1999), WSS less than 1.5 Pa leads to atherosclerotic plaque development and progression. From Fig. 4, 5 and 6, it is found that WSS areas having less than $1.5 \mathrm{~Pa}$ after the stenosis $70 \% \mathrm{AS}$ decreases with the increase in severity(90\%AS), which is consistent with the studies published(Buradi and Mahalingam 2018). The higher TAWSS in the range between 0.13 and 0.15 was noted across the stenosis. Whereas the low TAWSS region in the range between $0 \mathrm{~Pa}$ to $0.03 \mathrm{~Pa}$ was found. For the models P70_D70 and P70_D90 the low regions of TAWSS were found between the stenosis and after the distal stenosis due to protuberance because of the low blood flow. The oscillatory shear index (OSI) characterizes the variation in the direction of the WSS vector from a prime blood flow direction during the cardiac cycle. The higher magnitude of OSI causes significant damage to the endothelial cell layers by severe stretching and compression of this layer results in the rupture of plaque as reported by several studies. It is clearly seen in Fig. 10, 11, and 12 that the higher OSI values at the shoulder of the stenosis and it is increased for the $90 \%$ AS. The increase in the OSI was found in the range 0.38 to 0.4 , Also, a slightly increase in area of OSI was observed at the distal stenosis for the models P70_D90 and P90_D70 as compared to the model P70_D70. This study could improve our clinical comprehension of blood flow and its impact due to multi stenosis disease, which could lead to the development and progression of atherosclerosis. There was some limitation of this study which should be addressed. The current study morphology of stenosis investigated was considered axisymmetric for all the models as it is believed that the stenosis does not have a particular shape reported by several studies. There the actual stenoses models should be considered in future studies. In this study, the idealized left coronary artery models have been developed instead of considering the patient-specific left coronary artery model; therefore, the simulated results do not completely replicate the actual physiological situation. An idealized model's wall was considered rigid rather than a moving wall in the current study, which does not represent the real physiological situation.

\section{CONCLUSION}

Following conclusions are drawn from this study.

- The low wall shear stress area increases as the interspacing distance between stenosis is small for the model P70_D70.

- It is found that a larger area of low wall shear stress was observed for the model P70_D70 as compared to the other two models investigated.

- The rupture of stenosis risk increases for the model P90_D70 as compared to P70_D70 and P70_D90 models.

- The hemodynamic parameters WSS, TWSS, and OSI significantly affected the severity of stenosis and interspacing distance between the stenosis.

- This study further improves the understanding of the progression and development of stenosis and plaque rupture. 


\section{ACKNOWLEDGEMENTS}

The author extends his appreciation to the Deanship of Scientific Research at King Khalid University for funding this work through the research groups program under grant number RGP.1/327/42.

\section{REFERENCES}

Badruddin, I. A., S. Kamangar, A. Algahtani, M. A. Khan, S. A. NJ, C. A. Saleel and T. Y. Khan (2019). A Computational Study of Curvature Effect on the Coronary Diagnostic Parameters in Stenosed Coronary Artery. Chinese Journal of Mechanical Engineering 40(5), 539-546.

Banerjee, R. K., L. H. Back, M. R. Back and Y. I. Cho (2003). Physiological flow analysis in significant human coronary artery stenoses. Biorheology 40(4), 451-476.

Berger, S. and L. D. Jou (2000). Flows in stenotic vessels. Annual Review of Fluid Mechanics 32(1), 347-382.

Buradi, A. and A. Mahalingam (2018) Effect of Stenosis Severity on Wall Shear Stress Based Hemodynamic Descriptors using Multiphase Mixture Theory, Journal of Applied Fluid Mechanics 11(6), 1497-1509.

Caro, C., J. Fitz-Gerald and R. Schroter (1969) Arterial wall shear and distribution of early atheroma in man. Nature 223(5211), 11591161.

Chatzizisis, Y. S., A. U. Coskun, M. Jonas, E. R. Edelman, C. L. Feldman and P. H. Stone (2007). Role of endothelial shear stress in the natural history of coronary atherosclerosis and vascular remodeling: molecular, cellular, and vascular behavior. Journal of the American College of Cardiology 49(25), 2379-2393.

Dash, R. K., Jayaraman, G. and K. N. Mehta (1999). Flow in a catheterized curved artery with stenosis. Journal of Biomechanics 32(1), 49-61.

Frauenfelder, T., E. Boutsianis, T. Schertler, L. Husmann, S. Leschka, D. Poulikakos and H. Alkadhi (2007). Flow and wall shear stress in end-to-side and side-to-side anastomosis of venous coronary artery bypass grafts. Biomedical Engineering Online 6(1), 35.

Govindaraju, K., G. N. Viswanathan, I. A. Badruddin, S. Kamangar, N. S. Ahmed and A. A. Al-Rashed (2016). A parametric study of the effect of arterial wall curvature on non-invasive assessment of stenosis severity: computational fluid dynamics study. Current Science 483-491.

He, X. and Ku, D. N. (1996). Pulsatile flow in the human left coronary artery bifurcation: average conditions. Journal of Biomechanical Engineering 118(1), 74-82.

Jahangiri, M., M. Saghafian and M. R. Sadeghi (2015). Numerical simulation of hemodynamic parameters of turbulent and pulsatile blood flow in flexible artery with single and double stenoses. Journal of Mechanical Science and
Technology 29(8), 3549-3560.

Kamangar, S., G. Kalimuthu, I. Anjum Badruddin, A. Badarudin, N. J. Salman Ahmed and T. M. Khan (2014). Numerical investigation of the effect of stenosis geometry on the coronary diagnostic parameters. The Scientific World Journal 2014, 1-7.

Kamangar, S., I. A. Badruddin, N. A. Ahamad, K. Govindaraju, N. Nik-Ghazali, N. J. Ahmed and T. M. Khan (2017a). The Influence of Geometrical Shapes of Stenosis on the Blood Flow in Stenosed Artery. Sains Malaysiana 46(10), 1923-1933.

Kamangar, S., I. A. Badruddin, K. Govindaraju, N. Nik-Ghazali, A. Badarudin, G. N. Viswanathan and T. Y. Khan (2017b). Patient-specific 3D hemodynamics modelling of left coronary artery under hyperemic conditions. Medical \& Biological Engineering \& Computing 55(8), 1451-1461.

Kamangar, S., N. J. Salman Ahmed, I. A. Badruddin, N. Al-Rawahi, A. Husain, K. Govindaraju and Y. Khan (2019). Effect of stenosis on hemodynamics in left coronary artery based on patient-specific CT scan. Bio-Medical Materials and Engineering 30(4), 463-473.

Karayannacos, P. E., N. Talukder, R. M. Nerem, S. Roshon and J. S. Vasko (1977). The role of multiple noncritical arterial stenoses in the pathogenesis of ischemia. The Journal of Thoracic and Cardiovascular Surgery 73(3), 458-469.

Karimi, S., M. Dabagh, P. Vasava, M. Dadvar, B. Dabir and P. Jalali (2014). Effect of rheological models on the hemodynamics within human aorta: CFD study on CT image-based geometry. Journal of Non-Newtonian Fluid Mechanics 207, 42-52.

Konala, B. C., A. Das and R. K. Banerjee (2011). Influence of arterial wall-stenosis compliance on the coronary diagnostic parameters. Journal of Biomechanics 44(5), 842-847.

Li, M. X., J. J. Beech-Brandt, L. R. John, P. R Hoskins and W. J. Easson (2007). Numerical analysis of pulsatile blood flow and vessel wall mechanics in different degrees of stenoses. Journal of Biomechanics 40(16), 3715-3724.

Malek, A.M., S.L. Alper and S. J. J. Izumo (1999). Hemodynamic shear stress and its role in atherosclerosis 282(21), 2035-2042.

Moreno, C. and K. Bhaganagar (2013). Modeling of Stenotic coronary artery and implications of plaque morphology on blood flow. Modelling and Simulation in Engineering 2013.

Nosovitsky, V. A., O. J. Ilegbusi, J. Jiang, P. H. Stone and C. L. Feldman (1997). Effects of curvature and stenosis-like narrowing on wall shear stress in a coronary artery model with phasic flow. Computers and Biomedical Research 30(1), 61-82. 
S. Kamangar / JAFM, Vol. 15, No. 1, pp. 15-23, 2022.

Rathish Kumar, B. V., T. Yamaguchi, H. Liu and R. Himeno (2002). A numerical study of an unsteady laminar flow in a doubly constricted 3D vessel. International Journal for Numerical Methods in Fluids 38(12), 1159-1176.

Stone, P. H., A. U. Coskun, Y. Yeghiazarians, S. Kinlay, J. J. Popma, R. E. Kuntz and C. L. Feldman (2003). Prediction of sites of coronary atherosclerosis progression: in vivo profiling of endothelial shear stress, lumen, and outer vessel wall characteristics to predict vascular behavior. Current Opinion in Cardiology 18(6), 458-470.

Suess, T., J. Anderson, L. Danielson, K. Pohlson, T.
Remund, E. Blears and P. Kelly (2016). Examination of near-wall hemodynamic parameters in the renal bridging stent of various stent graft configurations for repairing visceral branched aortic aneurysms. Journal of Vascular Surgery 64(3), 788-796.

Wu, J., G. Liu, W. Huang, D. N. Ghista and K. K. Wong (2015). Transient blood flow in elastic coronary arteries with varying degrees of stenosis and dilatations: CFD modelling and parametric study. Computer Methods in Biomechanics and Biomedical Engineering 18(16), 1835-1845. 